\title{
Investigation on Regional Recognition of Open Access Journals
}

\author{
Li Shen \\ Library, Harbin Institute of Technology \\ Harbin Institute of Technology, HIT \\ Harbin, China \\ lishen@hit.edu.cn \\ * Corresponding Author \\ Zhang Wanfeng * \\ Harbin Environmental Monitoring Center \\ Harbin Environmental Monitoring Center \\ Harbin, China \\ zhangwanfeng1977@126.com \\ * Corresponding Author
}

\author{
Yu Guang \\ Library, Harbin Institute of Technology \\ Harbin Institute of Technology, HIT \\ Harbin, China \\ yug@hit.edu.cn
}

\begin{abstract}
To investigate regional differences in open access among China, USA and Japan, we presented different test methods in contrast distributions of open access journals in SCI-E. We analyzed quartiles in category and influence of open access journals in basic subjects (mathematics, physics, chemistry) by JCR, found top $0.10 \%$ papers and highly cited papers according to baselines in ESI. The results show that Chinese scholars approved open access journals better than USA and Japan, the ratio is $6.78 \%$; three countries have significant difference in $\mathrm{OA}$ overall distribution; $\mathrm{OA}$ articles of China increases significantly since 2008, while Japan is relatively stable growth in mathematics, fluctuating in physics and chemistry; three countries publish more than $30 \%$ in top $50 \%$ journals in the each subject; Chinese scholars issue more than $50 \%$ papers in open access journals of chemistry and mathematics; highly cited papers are mostly interdisciplinary research papers or publishing in interdisciplinary journals. Acceptances to OA journals seem likely to incline to some publisher's country/territory.
\end{abstract}

Keywords- Open Access; regional recognition; highly cited; Kruskal-Wallis test; Wilcoxon test

\section{INTRODUCTION}

Open access (OA) movement began in the 1990s. OA repository arXiv first appeared on the Internet in 1991. Its intention is to solve the current "crisis of scholarly journal publishing" to promote free access in Internet, dissemination of scientific research, academic exchange and information publication, and ensure long-term preservation of scientific information ${ }^{[1]}$. The famous international Open Access Journals Directory DOAJ (Directory of Open Access Journals) only includes 375 kinds of peer-reviewed OA journals in $2003^{[2]}$, the number changes into 10,294 till February 2015, covers almost all of the disciplines, and promotes the free exchange of scholarly information, and maximize the impact of scientific research ${ }^{[3]}$. Users reduce to rely on Library Information Resources in OA environment. OA publishing model presents a challenge to journal management of the traditional library ${ }^{[4]}$. To understand the real quality of OA

\author{
Tan Shuang \\ Harbin University of Science and Technology \\ Harbin University of Science and Technology, \\ HRBUST \\ Harbin, China \\ tanshuang@hrbust.edu.cn
}

resources, integrate OA resources for library, it becomes new challenges for library. At present, researchers focused on open access journals in ISI and other platforms (database) to find the status quo of open access journals in one disciplinary ${ }^{[5,6]}$; OA academic publishing features ${ }^{[7,8]}$; OA publishing how to affect the citation index, evaluation and control of OA quality ${ }^{[9-16]}$; assessment of university research in network by OA ${ }^{[17]}$; researchers accept open access to what degree ${ }^{[18-20]}$; OA data format conversion, data download, etc. [21-24]; OA journals distribution, acquisition mode, use status, trends, comparison in different countries ${ }^{[25-38]}$, etc. . In order to investigate regional differences in open access among China, USA and Japan, we chose SCI-E and used hypothesis test method, collected data of open-access journals and nonopen access journals; compared the main research fields among China, Japan, and the United States to explore the similarity and differences in subject distribution of open access and non-open access papers; contrast differences among basic subjects (mathematics, physics, chemistry); analyzed main journals that three countries' scholars published their articles most in three subjects, and evaluated journals' quartile and impact in category according to JCR; at the same time, analyzed Top $0.10 \%$ highly cited papers (open access vs. non open access) based on baseline in ESI.

\section{Methodology}

Because SCI-E began to identify open access and non open access by refining results from 2014, therefore, we used the function to classify the data directly. We counted all published data in basic subjects (mathematics, physics, chemistry) and data from China, USA, Japan in 1985 to 2014 (retrieving time ended in 02/04/2015). And we used hypothesis method and other statistical method in analyzing data. 


\section{COMPARSION AND ANALYSIS}

\section{A. Comparison of published papers in China, USA, Japan}

All three countries published papers as shown in TABLE I.The three countries published papers mainly in non open access journals, and Chinese scholars had the highest recognition of open access journals, $6.78 \%$ of their articles were published in open access journals, while the USA scholars only published $2.49 \%$ in OA journals. At the same time, we studied main research directions in open access, non open access, and total of three counties. We found the research directions are same in non open access and total published, non open access papers controlled major research direction. The first three research directions are chemistry, physics and engineering in China and the USA, while they are physics, chemistry, and engineering in Japan. Three countries' main research directions are apparently different in open access. Only three basic subjects of China are among the top 10, in which mathematics (13018 articles, top2), chemistry (7472 articles, top 4), physics (4855 articles, top10); Japan has two subjects into the top 10, physics (6894 articles, top4), chemistry (5848 articles, top5); and the United States only physics (8280 articles, top9) into the top 10 . In physics the United States has contributed the most to global articles output(712,855), Japan $(328,507)$ Second, China $(296,836)$ Third, the three countries accounted for $46.58 \%$ of all the published papers in this subject; in chemistry USA $(879,299)$ first , China $(377,744)$ Second, Japan $(297,518)$ Third, the three countries accounted for $45.53 \%$ of all the published papers in this subject; in mathematics USA $(278,410)$ first, China $(115,064)$ Second, Japan $(43,459)$ Eighth, the three countries accounted for $44.23 \%$ of all the published papers in this subject. Therefore, we choose these three countries in our study.

TABLE I CHINA USA AND JAPAN PUBLISHED ARTICLES IN OPEN ACCESS AND NON OPEN ACCESS JOURNALS OF SCI-E

\begin{tabular}{cccc}
\hline Country & Articles in OA & Articles in non OA & $\begin{array}{c}\text { Total } \\
\text { articles }\end{array}$ \\
\hline China & 129751 & 1784963 & 1914714 \\
USA & 239372 & 937105 & 9610477 \\
Japan & 105968 & 2133282 & 2239240 \\
\hline
\end{tabular}

B. Comparative study on open access journals in basic subjects among China, USA, and Japan in 2005-2014

We used multiple independent sample test KruskalWallis $H(K)$, respectively, in open access papers test of China, USA, and Japan from 2005 to 2014 among physics, chemistry, and mathematics (results in Table 2). The results indicated that three countries had significant difference in OA distribution, and there was significant difference in physics, mathematics.

For a more objective analysis of scholars' recognitions in three countries to open access, we let open access papers and non open access papers as a ratio (percentage) in three countries. The results showed that the trends were overall upward of physics, chemistry, mathematics in China and USA, and China increased significantly since 2008, while Japan was relatively stable growth in mathematics, and the others were fluctuating apparently.
TABLE II . KRUSKAL-WALLIS H TEST OF OA DISTRIBUTION IN BASIC SUBJECTS

\begin{tabular}{cccc}
\hline country & physics & chemistry & mathematics \\
\hline China(Mean Rank) & 15.50 & 20.55 & 20.20 \\
USA(Mean Rank) & 21.60 & 13.70 & 19.60 \\
Japan(Mean Rank) & 9.40 & 12.55 & 6.70 \\
N & 10 & 10 & 10 \\
Chi-square & 9.603 & 5.073 & 15.015 \\
Df & 2 & 2 & 2 \\
Asymp.sig & .008 & 0.079 & .001 \\
\hline
\end{tabular}

C. Analysis of Open Access Journals in China, USA and Japan (according to JCR)

We statistic the top ten journals that published open access papers most in physics, chemistry, mathematics of three countries (impact factor on the basis of 2013 JCR), the results were as follows: in physics there were three journals in Q1 (Quartile in Category), one in Q2, journals that published papers most were all in Q1 quartile of different branches in physics, and China had absolute advantage in journal "NANOSCALE RESEARCH LETTERS"; in chemistry there were three journals in Q2, Chinese scholars published more than $50 \%$ papers in the first three journals that published the most papers in chemistry; in mathematics three journals were in Q1, three journals in Q2 (we chose the highest quartile of each journal), Chinese scholars published more than $88 \%$ in the first three journals. And, by the impact factor trend of JCR we found just as qin ${ }^{[10]}$ said that open access journals' impact factors didn't continue increasing and impact factors of OA journals were not higher than the non $\mathrm{OA}$ journals. Meanwhile, the acceptances of open access journals had something with journals' country/territory. As we saw PROGRESS OF THEORETICAL PHYSICS which published the most papers in physics, although published in English, the publisher was C / O KYOTO UNIV (Japan). Therefore, it mainly collected papers from Japan, 4445 papers were completed by the Japanese authors (total papers were 5019).

\section{Comparisons in open access journals with non open access journals in basic subjects}

1) Total comparison in open access journals with non open access journals in basic subjects

We used the Wilcoxon test and Sign test for paired samples as open access articles and non open access articles of 2005-2014 in physics, chemistry, mathematics for Nonparametric tests. The results showed that the overall distribution between open access and non-open access journals in the three subjects were significantly different (Wilcoxon test sig=0.005, Sign test sig=0.002). Major open access journals in physics were 49, while non open access journals were 1187 (the former: the latter was 1:24.22); major open access journals in chemistry were 52, while non open access journals were 1610 (the former: the latter was 1:30.96); major open access journals in mathematics were 83 , while non open access journals were 1311 (the former: the latter was 1: 15.80). 
2) Top $0.10 \%$ articles contrast in basic subjects

TABLE III TOP 0.10\% ARTICLES BETWEEN OPEN ACCESS JOURNALS AND NON OPEN ACCESS JOURNALS OF 2005-

\begin{tabular}{|c|c|c|c|c|c|c|c|c|c|c|c|}
\hline Subject & year & 2005 & 2006 & 2007 & 2008 & 2009 & 2010 & 2011 & 2012 & 2013 & 2014 \\
\hline \multirow[t]{4}{*}{ Physics } & Top $0.10 \%$ citations & 507 & 428 & 396 & 417 & 310 & 260 & 174 & 132 & 56 & 14 \\
\hline & Total articles & 124 & 134 & 143 & 122 & 155 & 165 & 195 & 191 & 273 & 410 \\
\hline & Non OA articles & 123 & 134 & 143 & 121 & 152 & 163 & 195 & 191 & 272 & 403 \\
\hline & OA articles & 1 & 0 & 0 & 1 & 3 & 2 & 0 & 0 & 1 & 7 \\
\hline \multirow[t]{4}{*}{ Chemistry } & Top $0.10 \%$ citations & 507 & 481 & 450 & 442 & 362 & 320 & 209 & 156 & 64 & 17 \\
\hline & Total articles & 156 & 144 & 166 & 169 & 218 & 225 & 270 & 251 & 388 & 527 \\
\hline & Non OA articles & 156 & 144 & 166 & 169 & 362 & 225 & 269 & 250 & 388 & 523 \\
\hline & OA articles & 0 & 0 & 0 & 0 & 0 & 0 & 1 & 1 & 0 & 4 \\
\hline \multirow[t]{4}{*}{ Mathematics } & Top $0.10 \%$ citations & 176 & 162 & 143 & 132 & 97 & 76 & 52 & 31 & 16 & 7 \\
\hline & Total articles & 119 & 96 & 107 & 98 & 129 & 130 & 142 & 181 & 185 & 122 \\
\hline & Non OA articles & 116 & 94 & 103 & 95 & 126 & 123 & 134 & 167 & 163 & 114 \\
\hline & OA articles & 3 & 2 & 4 & 3 & 3 & 7 & 8 & 14 & 22 & 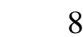 \\
\hline
\end{tabular}

According to per year cited indicators data of Baseline database based on ESI, we analyzed statistically top $0.10 \%$ articles of $2005-2014$ among the three basic subjects. The results indicated the top $0.10 \%$ articles primarily published in non open access journals, open access journals in mathematics had more top $0.10 \%$ articles than in physics and chemistry (as shown in Tab.3).

3) Contrast highly cited papers between open access and non open access journals

We examined highly cited papers between open access journals and non open access journals respectively in physics, chemistry, and mathematics during 2005-2014. The highest cited paper in open access journals was Sakai, T, et al.. 2005 "Low energy hadron physics in holographic QCD" published in Progress of Theoretical Physics (PHYSICS, MULTIDISCIPLINARY Q1, impact factor 2.056, journals ranking 18), which was cited 679; the most cited non open access journal article was Geim, AK, et al. published in Nature Materials 2007 (PHYSICS, APPLIED Q1, PHYSICS, CONDENSED MATTER Q1, impact factor 36.425, journals ranking 1) "The rise of graphene", citations were 11639. The highest cited article in open access journal of chemistry was Bai, Hua et al. published in Sensors 2007(CHEMISTRY, ANALYTICAL Q2, journals ranking 36, ELECTROCHEMISTRY Q3 journals ranking 15, INSTRUMENTS \& INSTRUMENTATION Q1 journals ranking 10, impact factor 2.048) "Gas sensors based on conducting polymers", had been cited 365 times; the most cited non open access journal article was Geim, AK, et al. 2007 published in Nature Materials (CHEMISTR Y, PHYSICAL Q1, MATERIALS SCIENCE, MULTIDISCIPLINARY Q1, impact factor 36.425, journals ranking 1) "The rise of grapheme", 11,639 citations. The most cited open access journal article in mathematics was Friedman, Jerome, et al., published in 2010 Journal of Statistical Software (STATISTICS \& PROBABILITY Q1 journals ranking 2, COMPUTER SCIENCE, INTERDISCIPLINARY APPLICATIONS Q1 journals ranking 9, impact factor 3.801) "The Regularization Paths for Generalized Linear Models via Coordinate Descent", 659 citations; The most cited non open access journal article was Larkin, MA, et al. published in Bioinformatics (MATHEMATICAL \& COMPUTATIONAL BIOLOGY Q1 journals ranking 4, BIOCHEMICAL RESEARCH METHODS Q1 journals ranking 12, BIOTECHNOLOGY \& APPLIED MICROBIOLOGY Q1 journals ranking 18, impact factor 4.621) "Clustal W and Clustal $\mathrm{X}$ version 2.0", 9012 citations. These highly cited papers were published in a professional journal and their quartiles in category were Q1. Interdisciplinary research or articles published in interdisciplinary journals were easy to widely cite.

In general, there are significant differences in the distribution between open access journals and non open access journals of the three basic subjects; Top $0.10 \%$ articles are major in non open access journals; the highest cited papers in open access journals and non open access journals were published in professional journals in Q1 quartile (that is, top $25 \%$ of journals in category), and these highly cited papers, mostly were interdisciplinary research papers or published in the interdisciplinary journals.

\section{CONCLUSION}

Through comparative analysis of open access and non open access journals in physics, chemistry, and mathematics (three basic subjects), we found that Chinese scholars would like to publish their research in open access journals, the ratio was $6.78 \%$; three countries had significant difference in OA distribution in three subjects, and there were significant difference in physics, mathematics; China and USA both increased in three subjects by the ratio of open access and non open access journals, and China increased significantly since 2008, while Japan was relatively stable growth in mathematics, fluctuating in chemistry and physics; the proportion of open access journals to non open access journals were 1:24.22 in physics, 1:30.96 in chemistry, 1:15.80 in mathematics, there were significant differences of open access journals and non open access journals in overall distribution of the three basic subjects; Top $0.10 \%$ papers mainly published in non open access journals; highly cited papers were published in professional journals in the Q1 quartile (that is, top $25 \%$ of their subject), and these highly cited papers, mostly were research papers cross different subject or published in interdisciplinary journals. The results showed that: open access journals in SCIE were still a small part, three countries published more than $30 \%$ in top 50\% open access journals of each subject. Chinese scholars issued more than $50 \%$ papers in open access journals published in chemistry and mathematics. Highly cited papers, mostly were interdisciplinary research papers or published in the interdisciplinary journals. Acceptances of open access journals seemed likely to incline to some country/territory (eg. PROGRESS OF THEORETICAL PHYSICS). The United States and Chinese scholars would like to issue in open access journals, they would lead to more widely cited of open access journals as major 
countries in basic research fields. So they would promote the development of open access journals. Meanwhile, we hope open access journals in China would continuously improve the quality to meet the scientific research needs of Chinese scholars.

\section{ACKNOWLEDGMENT}

This article is supported by Heilongjiang Art Planning Project(2015D087)

\section{REFERENCES}

[1] Interactive Encyclopedia. Open access journal [EB/OL]. (2012-0904)[2015-01-20].

http://www.baike.com/wiki/\%E5\%BC\%80\%E6\%BA\%90\%E6\%9 C\%9F\%E5\%88\%8A.

[2] Han Jian, Zhang Jingjing, Huang Heqing. The situation of open access in SCI 2010 pharmacological /pharmaceutical professional periodicals[J]. Chinese Journal of Scientific and Technical Periodicals, 2012(05):763-766.

[3] Wang Hongping. A current research on the construction of the economic OAJ Resources[J]. Journal of Modern Information, 2013(08):127-131.

[4] Liu Xiaoji, Cao Chunxiang. The influence and application of open access journals on academic libraries' journals work[J].Library Work in Colleges and Universities, 2012(05).

[5] Pelizzari E. Academic authors and open archives: A survey in the social science field[J]. LIBRI, 2004,54(2):113-122.

[6] Saberi M K, Abedi H. Accessibility and decay of web citations in five open access ISI journals[J]. Internet Research, 2012,22(2):234-247.

[7] Kousha K. Characteristics of open access scholarly publishing A multidisciplinary study[J]. ASLIB Proceedings, 2009,61(4):394406.

[8] Laakso M. Green open access policies of scholarly journal publishers: a study of what, when, and where self-archiving is allowed[J]. Scientometrics, 2014,99(2):475-494.

[9] Qin Wenzhen, Liu Shurui, Xiao Qiong. Comparable analysis on the applications of quality evaluation methods for open access journals[J]. Sci-Tech Information Development \& Economy, 2013(06):6-8.

[10] Qin Xiaochuan, Jiang Shiqing, Liu Xueli. Effect of relevant Citation Index of OA on China's academic journals published[J]. Chinese Journal of Scientific and Technical Periodicals, 2014(08):985-989.

[11] Ku Liping, Zhang Xiaolin, Chu Jingli, Li Lin, Zeng Yan. Evaluation and selection for open access journal: quality, openness and service abilities[J]. Library and Information Service, 2013(01):49-54.

[12] Qin Wenzhen, Xiao Qiong. Application of impact factor in evaluation of Open Access Journals[J]. View on Publishing, 2013(04):68-69.

[13] Academic quality control of Open Access Journals: the surprising results [J]. Chinese Archives of General Surgery(Electronic Version), 2013(06):519.

[14] Su Xiaobo. Analysis of open access journal quality control and copyright protection measures[J]. Heilongjiang Science, 2013(12):86-87.

[15] Koler-Povh T, Juznic P, Turk G. Impact of open access on citation of scholarly publications in the field of civil engineering[J]. Scientometrics, 2014,98(2):1033-1045.

[16] Singh N, Chikate A. Open access LIS periodicals and digital archives An evaluation with reference to Asian countries[J]. Electronic Library, 2014,32(5):710-725.
[17] Fan Wenqiang, Liu Qinghui. Web - influence evaluation research achievements of university from view of open access: Taking " 985 " universities as the object of study[J]. Journal of Intelligence, 2014(04):35-40.

[18] Shi Lei, Zhai Zhonghui. Construction of influencing factor model of researcher's acceptance of OA journals[J]. Information Research, 2015(01):26-28.

[19] Zhai Zhonghui, Shi Lei. Evidential analysis of the factors influencing researchers' adoption of OA journals[J]. Journal of Academic Library and Information Science, 2014(02):5-8.

[20] Su Xiaobo. Analyzing the factors influencing researchers' acceptance of open access journal publishing from the angle of knowledge sharing[J]. Library, 2014(05):23-27.

[21] Ma Qi, Wang Shan, Zhang Weimin, Li Peng. Open Access data format conversion method in practice[J]. Heilongiiang Science and Technology Information, 2013(30):156-157.

[22] Yang Zhenxiong, Cai Zurui, Chen Guohua, Tang Yong, Zhang Long. Design and implementation of distributed web crawler for open access journal[J]. Journal of Frontiers of Computer Science and Technology, 2014(10): 1187-1194.

[23] Yamamoto Y, Yamaguchi A, Yonezawa A. Building Linked Open Data towards integration of biomedical scientific literature with DBpedia[J]. Journal of Biomedical Semantics, 2013,4(8).

[24] Peset F, Ferrer-Sapena A, Subirats-Coll I. Linked open data and Open data. Its impact in the field of libraries and information science[J]. Professional de la Infromacion, 2011,20(2):165-173.

[25] Liu Wanping, Sun Bo. A comparative study on open access between China and India[J]. Library and Information Service, 2013(07):65-69.

[26] Dong Yue. Study on the development trends of OA journals based on its review and current status analysis[J]. Agriculture Network Information, 2013(08):45-48.

[27] Sun Shaoju. Analysis and research on 38 open access journals resources of library and information science and their acquisition modes[J]. Library Work in Colleges and Universities, 2013(02):6970.

[28] Li Si. Status quo of open access in developing countries[J]. Chinese Journal of Medical Library and Information Science, 2014(07):59-63.

[29] Liao Siqin, Zhou Yu, Wei Tailiang. Empirical research on the utilization situation of open access journals of libraries in China[J]. Library Development, 2014(12):22-25.

[30] Zhang Wei. A review of research on open access in China in the last five years[J]. Journal of Baoding University, 2014(05):97-102.

[31] Li Wu, Liang Xiaojian, Yang Lin. International progress in recent five years of open access movement [J]. Science-Technology \& Publication, 2013(08):71-75.

[32] Feng Qing, Zhao Ying, Wan Yan. Research on the utilization of open access journals[J]. Library Tribune, 2013(01):173-176.

[33] Li Si. A comparative study of China, Japan and South Korea in open access[J]. Research on Library Science, 2014(04):22-26.

[34] Gong Weizhong, Yang Yang. Comparison of China and foreign in open access journal publishing[J]. View on Publishing, 2014(13):59-61.

[35] Sotudeh H, Horri A. Countries positioning in open access journals system: An investigation of citation distribution patterns[J]. Scientometrics, 2009,81(1):7-31.

[36] Simek P, Jarolimek J, Cerna E, et al. Integration of a Scientific and Scholarly Research Journal's Content into the Central International Repository[J]. Agrarian Perspectives, 2011:415-422.

[37] McMullan E. Open access mandate threatens dissemination of scientific information[J]. Journal of Neuro-ophthalmology, 2008,28(1):72-74.

[38] Bjork B, Solomon D. Open access versus subscription journals: a comparison of scientific impact[J]. BMC Medicine, 2012,10(73). 